\title{
Paciente VIH positivo con tuberculosis sistémica y lesiones osteolíticas de la calvaria, semejando mieloma múltiple.
}

\author{
Patient HIV positive with systemic tuberculosis and osteolytic calvarial lesions mimicking mul- \\ tiple myeloma
}

\author{
Victor Mechán ${ }^{1,2}$, Antonio Salas ${ }^{3}$, Yuri García ${ }^{4}$, Félix Llanos ${ }^{5}$, Jorge Cornejo ${ }^{6}$, Rocío Bringas \\ ${ }^{1}$ Servicio de Hematología, Hospital Nacional Dos de Mayo, Lima, Perú \\ ${ }^{2}$ Docente de Medicina, Universidad Nacional Mayor de San Marcos, Lima, Perú \\ ${ }^{3}$ Servicio de Neumología, Hospital Nacional Dos de Mayo, Lima, Perú \\ ${ }^{4}$ Servicio de Enfermedades Infecciosas y Tropicales, Hospital Nacional Dos de Mayo, Lima, Perú \\ ${ }^{5}$ Medico Asistente de Neumología, Hospital Nacional Dos de Mayo, Lima, Perú \\ ${ }^{6}$ Médico Residente de Neumología. Hospital Nacional Dos de Mayo, Lima, Perú \\ ${ }^{7}$ Alumna, $6^{\circ}$ año de Medicina, Universidad Nacional Mayor de San Marcos, Lima, Perú
}

\begin{abstract}
Resumen
Varón de 33 años, soltero, sin ocupación fija, promiscuo, con historia de relaciones homosexuales, ingesta crónica de alcohol, consumo de tabaco, marihuana, pasta básica y clorhidrato de cocaína, desde los 18 años. Infectado por el virus de la inmunodeficiencia humana, recibía tratamiento irregular con terapia antirretroviral de gran actividad (Targa) entre 2006 y 2008, regularizada en los últimos 12 meses. Por presentar bacilo ácido alcohol resistente (BAAR) pansensible en esputo, inició en junio 2008 tratamiento antituberculoso [2RHZE/4(HR)2)], retirándosele la medicación 6 meses después por presentar BAAR pulmonar negativo. En marzo de 2009, percibió dolor lumbar intenso, dificultad para caminar, hipertrofia de ganglios cervicales, tos, fiebre. Un cultivo de secreción ganglionar cervical descubrió M. tuberculosis resistente a rifampicina. La imagenología mostró lesiones osteolíticas múltiples en cráneo y vértebras dorsolumbares y tumefacciones renitentes en cuero cabelludo, antebrazo derecho y parrilla costal izquierda. Tras descartarse mieloma múltiple y metástasis cancerosas, se añadió tratamiento antituberculosis multidrogo resistente al Targa, notándose dos meses después involución de las tumefacciones renitentes, de la fiebre y mejoría del estado general. Tras 16 meses de tratamiento supervisado, el paciente aumentó $7 \mathrm{~kg}$ de peso, habiendo desaparecido la sintomatología que presentaba. Se discute la fisiopatología de las lesiones osteolíticas cráneo-vertebrales en un paciente con coinfección por el virus de la inmunodeficiencia humana y tuberculosis.

Palabras clave: VIH; tuberculosis vertebral; tuberculosis ganglionar; tuberculosis pulmonar; tuberculosis resistente a multidrogas.
\end{abstract}

\begin{abstract}
This is the case of a 33 years old man with history of homosexual relations and heavy alcohol drinking. Tobacco, marijuana, cocaine, basic paste and cocaine hydrochloride consumption since age 18. Because of human immunodeficiency virus infection he received irregular treatment (2006-2008) with highly active antiretroviral therapy (HAAT), regulated in the past 12 months. As the patient showed positive resistant acid-fast bacilli he received since June 2008 standard antituberculous treatment [2RHZE/4(HR)2)] (R: rifampicin; H: isoniazid; Z: pirazinamide; E: ethambutol) that was withdrawn 6 months later when the patient showed pulmonary negative acid fast bacilli. In March 2009 he felt intense lumbar pain, difficulty in walking, hypertrophy of cervical lymph nodes, cough and fever. Cervical lymph node discharge culture disclosed M. tuberculosis resistant to rifampicin. Imagenology showed several lytic lesions in skull and thoraco-lumbar vertebrae; also renitent swellings in scalp, right forearm and left rib cage. After multiple myeloma and metastatic cancer were excluded, the patient received multidrug resistant tuberculosis treatment added to HAAT, showing two months later involution of renitent swellings, absence of fever and overall improvement. After 16 months of supervised treatment, the patient gained $7 \mathrm{~kg}$ and all symptomatology previously present dissappeared. We discuss the pathophysiology of craniovertebral osteolytic lesions in a patient with coinfection of human immunodeficiency virus and tuberculosis.
\end{abstract}

Key words: HIV; tuberculosis, spinal; lymph node; tuberculosis, pulmonary; tuberculosis, multidrug-resistant.

An Fac med. 2010;71(3):207-11

\section{INTRODUCCIÓN}

La tuberculosis de la calvaria representa 0,2 a $1,3 \%$ de todos los casos comunicados de tuberculosis esquelética, rareza explicada por el deficiente suministro linfático craneal ${ }^{(1)}$. No obstante, los informes de tuberculosis (TBC) craneal están aumentando en países en desarrollo, a causa de malnutrición, deficientes condiciones socioeconómicas e incremento de síndromes de inmunodeficiencia ${ }^{(2-6)}$.
Después que el bacilo tuberculoso viaja por vía hematógena, se aloja en porciones del hueso esponjoso y el diploe craneal. Su diseminación posterior depende de la virulencia del bacilo y la resistencia del huésped. La infección oblitera capilares y reemplaza trabéculas óseas por tejido de granulación. Por la resistencia de la duramadre a la penetración por el bacilo TBC se explica la mayor incidencia de lesiones extradurales ${ }^{(1)}$. Notificamos este caso de TBC sistémica (lesiones osteolíticas vertebrales, abscesos fríos prevertebrales y afectación tuberculosa pulmonar y ganglionar) y afectación de la calvaria (lesiones líticas en huesos frontoparietales), expresada por tumefacciones del cuero cabelludo y trayectos fistulosos ${ }^{(7-10)}$, haciendo énfasis en la necesidad de un alto índice de sospecha para reconocer casos de TBC cráneovertebral. El presente caso describe 
asimismo el curso clínico de un paciente con reinfección tuberculosa ganglionar, pulmonar y lesiones osteolíticas cráneovertebrales, infectado simultáneamente por virus de la inmunodeficiencia humana (VIH), quien tras recibir terapia antituberculosis multidrogo resistente (MDR-TB) y Targa durante 16 meses, mejoró notablemente.

\section{REPORTE DE CASO}

Varón de 33 años con historia de relaciones homosexuales, ingesta crónica de alcohol, consumo de tabaco, marihuana, pasta básica y clorhidrato de cocaína, desde los 18 hasta los 28 años. En 1995 fue reactivo a anticuerpos a Herpes zoster y en 1997 , con hemoglobina $14,7 \mathrm{~g} / \mathrm{dL}$, presentó tos y diarrea crónica y fue reactivo a anticuerpos a HIV 1-2; la radiografía de tórax anteroposterior fue normal. En 1999 fue negativo a bacilo ácido alcohol resistente (BAAR) en esputo. En 2005, la reacción en cadena de polimerasa (PCR)auramina para BAAR en orina y esputo fue negativa. En setiembre 2006, la carga viral (CV) a VIH dio 41611 copias/mL, CD4 $22 \mathrm{cel} / \mu \mathrm{L}$. Inició Targa el 7/10/06, con zidovudina (AZT), lamivudina (3TC) y nevirapina (NVP). Abandonó el tratamiento Targa el 20/11/06. En 2007 presentó sinusitis frontal y diarrea crónica (quistes de Cystoisospora belli), CV 841 600 c/mL, CD4 3 c/ $\mu \mathrm{L}$. Reinició Targa el 7/6/07, esquema AZT+3TC+EFV (Efavirenz). En la radiografía de tórax hubo incremento de la trama broncovascular parahiliar, hemoglobina 6,3 g/dL, prueba de Coombs negativa. La anemia mejoró con transfusiones. En octubre presentó herpes genital y abandonó Targa. A inicios de diciembre mostró tos, sibilantes en $1 / 3$ superior de hemitórax derecho, subcrépitos en tercio inferior de hemitórax izquierdo. En la radiografía de tórax había opacidad bilateral, en panal de abeja, incremento del volumen del $2^{\circ}$ y $3^{\circ}$ arco costal izquierdo y $2^{\circ}$ arco costal derecho; había obstrucción de senos frontales y etmoidales. En la radiografía baritada, las asas intestinales estaban dilatadas, con pliegues mucosos engrosados, a predominio del colon ascendente. Mostró herpes genital, hemoglobina 10,3 g/dL, discreta rigidez de nuca, líquido cefalorraquídeo color cristal de roca, a goteo rápido, $\mathrm{ADA}$ 1,23 U/L (N: hasta 9), tinta china negativo, proteínas $45 \mathrm{mg} / \mathrm{dL}$, Pandy negativo, leucocitos $1 / \mathrm{mL}$, presión de apertura 175 $\mathrm{mm}$ de agua. Existía sospecha de TBC meníngea, pulmonar e intestinal.

En febrero 2008 tenía $\mathrm{Hb}$ 12,7 g/dL y en mayo reingresó al Targa, esquema $\mathrm{AZT}+3 \mathrm{TC}+\mathrm{EFV}$ que mantuvo hasta el 2010. Pero, en junio 2008 presentó diarreas, vómitos y tos con expectoración oscura; $\mathrm{CV}<300 \mathrm{c} / \mathrm{mL}, \mathrm{CD} 471 \mathrm{c} / \mu \mathrm{L}$, $\mathrm{BAAR}++$ en esputo, radiografía de tórax con incremento de la trama vascular pulmonar, bilateral, y la radiografía de columna lumbosacra fue normal; $\mathrm{Hb}$ 6,8 $\mathrm{g} / \mathrm{dL}$, mielograma con hipercelularidad, reactiva a proceso infeccioso general, células plasmáticas $4 \%$. La anemia mejoró con transfusiones. En agosto, la prueba de sensibilidad a drogas antiTBC con método de proporciones fue sensible a INH/SM/EMB/RFP/PZA. Inició [2RHZE/4(HR) $)_{2}$ ], hasta fines de noviembre (R: rifampicina, $\mathrm{H}$ : isoniazida, Z: pirazinamida, E: etambutol). En diciembre se halló dedos en palillo de tambor, tos, fiebre, disminución de peso (15 kg, en últimos 3 meses), hipoalgesia e hipoestesia de rama izquierda del trigémino, la úvula y lengua desviadas a la derecha e izquierda, respectivamente; rigidez de nuca, Kernig positivo bilateral. Tenía dolor dorso-lumbar intenso, debilidad y dolor en miembros inferiores a predominio del derecho; fuerza muscular

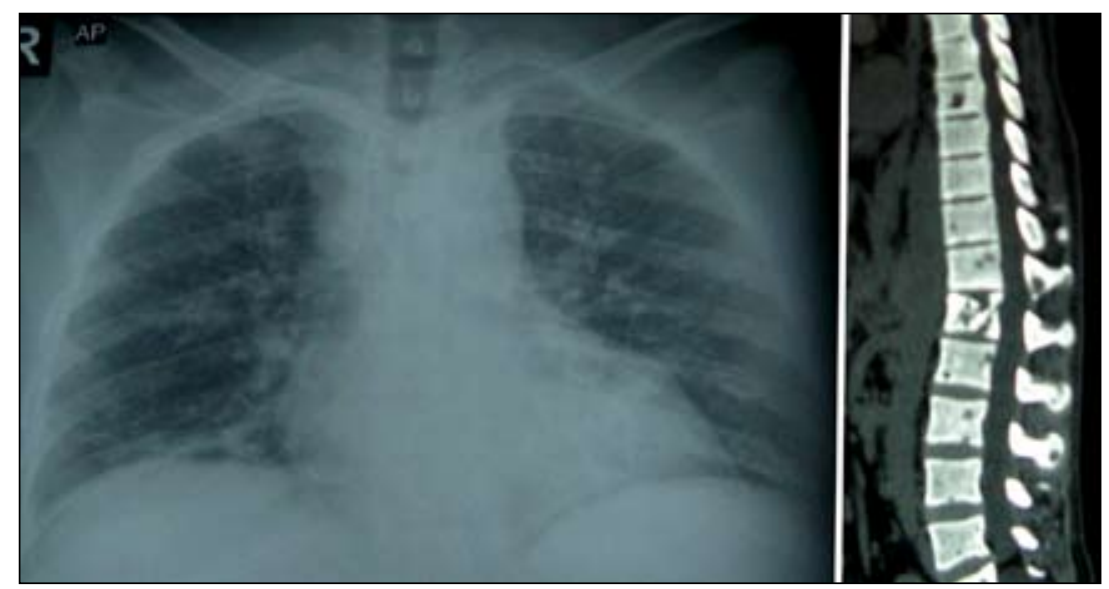

Foto 1. Radiografía de tórax muestra infiltrado pulmonar bilateral, cardiomegalia. Foto 2. Tomografia espiral multicorte de columna lumbosacra con lesiones osteolíticas en múltiples vértebras lumbosacras contiguas, sin comprometer los discos intervertebrales. 

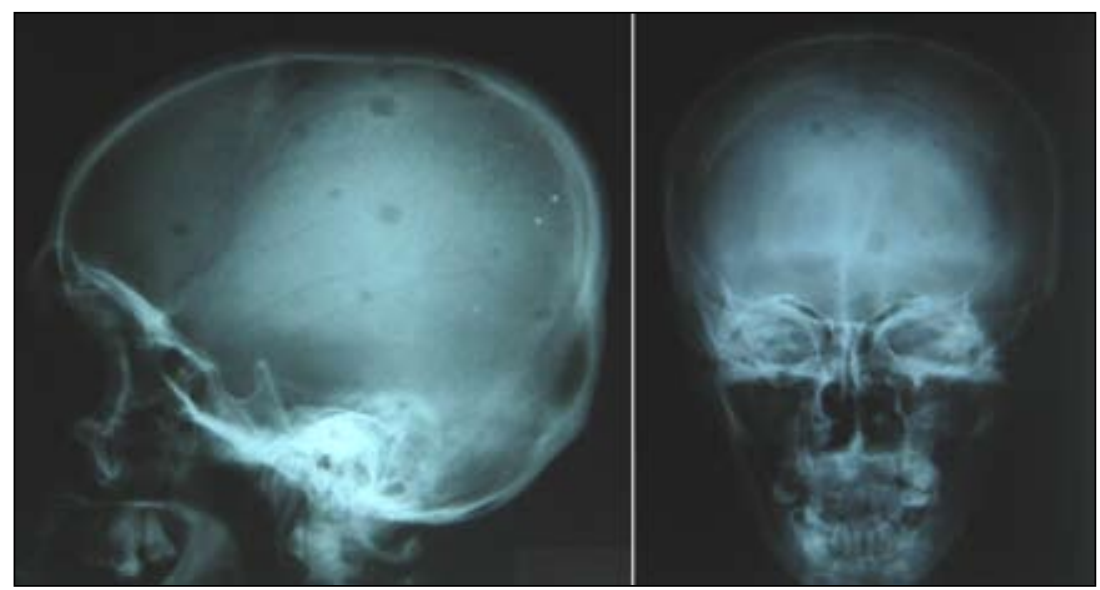

Foto 3. Vistas lateral y frontal de las radiografías simples de cráneo. Se observa múltiples lesiones osteolíticas en sacabocado, en áreas frontal, parietal y occipital. Lesión lítica parietal superior, con borde inferior esclerótico.

vitamina $\left.B^{6}(P I R)\right]$. En junio, el cultivo de ganglio en medio Lowenstein-Jensen mostró M. tuberculosis. El paciente fue internado en el Hospital Nacional Dos de Mayo, para determinar las causas de paraparesia y lesiones osteolíticas craneales.

En el examen físico en abril 2009, la presión arterial fue $100 / 80 \mathrm{mmHg}$, frecuencia cardiaca 120 por minuto, frecuencia respiratoria 26 por minuto, temperatura $38^{\circ} \mathrm{C}$; talla $1,59 \mathrm{~m}$, peso $60 \mathrm{~kg}$. La piel era pálida, con placas blanquecinas en el paladar duro. Había adenopatías cervical y axilar derechas, de 0,5 x 1 y 1 x $1 \mathrm{~cm}$, respectivamente. Se palpó dos tumefacciones renitentes en cuero cabelludo, una de $2 \mathrm{~cm}$ de diámetro en la parte media de la línea sagital craneal superior y otra de $0,5 \mathrm{~cm}$ de diámetro en área parietal izquierda. Existía una tumefacción renitente de 3 x $2 \mathrm{~cm}$, en el área externa del antebrazo derecho; la respiración era ruda en ambos pulmones, no se apreció choque de la punta cardiaco. Tenía lesiones micóticas en cintura abdominal, onicomicosis bilateral. El paciente estaba lúcido, orientado en tiempo, espacio y persona, bradipsíquico, obedecía las órdenes lentamente, Glasgow 15. No hubo signos meníngeos, pero sí paraparesia fláccida, con sensibilidad disminuida en miembros inferiores, hipopalestesia distal bilateral, hiperreflexia generalizada; el Hoffman estuvo presente bilateralmente. Existía hipotrofia muscular de los miembros in- feriores. Fue dado de alta en junio/2009. En setiembre 2009, en la consulta externa se detectó tumor renitente en la parrilla costal izquierda. El 22 de mayo 2010, CD4 tenía $129 \mathrm{cel} / \mu \mathrm{L}, \mathrm{CV}$ indetectable. El 20 de setiembre, CD4 $233 \mathrm{cel} / \mu \mathrm{L}, \mathrm{CV}$ indetectable. Durante sus últimos controles en la consulta externa de infectología, el paciente cursó asintomático, con exámenes hematológicos y bioquímicos normales.

En abril/2009, la Hb era 10,6 g/dL; la biopsia de ganglio cervical derecho fue BAAR positivo, auramina negativo, con presencia de granulomas tuberculosos; BAAR y cultivo de secreción de ganglio cervical mostró $\mathrm{M}$ tuberculosis resistente a 1,0 $\mu \mathrm{g} / \mathrm{mL}$ de RFP. En junio, BAAR en esputo negativo, cultivo de ganglio en Lowenstein Jensen positivo a $M$. tuberculosis, velocidad de sedimentación $125 \mathrm{~mm} / \mathrm{h}$. En radiografía de cráneo se vio lesiones líticas fronto-parieto-occipitales, $\mathrm{Hb} 10,1 \mathrm{~g} / \mathrm{dL}$, fosfatasa alcalina $299 \mathrm{UI}$ (N: 38 a 126), transaminasas oxal acéticas 42 UI (N: 21 a 56), oxal pirúvicas 10 UI (N: 21 a 70), proteínas totales $5 \mathrm{~g} / \mathrm{dL}$, albúmina $2,95 \mathrm{~g} / \mathrm{dL}(\mathrm{N}: 3,9 \mathrm{a}$ 4,6), seroglobulinas 2,05 g/dL (N: 0,6 a 1,5). El 16 de abril 2009, proteinograma electroforético en suero con albúmina 2,96 g/dL (N: 3,9 a 4,6), alfa-1 0,35 g/dL, alfa-2 0,91 g/dL, betaglobulina 0,94 g/dL, gammaglobulina 2,05 g/dL (N: 0,6 a 1,5); ausencia de componente monoclonal. Proteinograma electroforético en orina de 24 horas: ausencia de componente monoclonal. Segundo mielograma: hiperplasia de serie mieloide, a predominio de abastonados; $7 \%$ de células plasmáticas normales. En mayo 2009 radiografía de tórax con bronquiectasias e imágenes alveolares localizadas en segmentos 9 y 10 de pulmón derecho; atelectasias segmentarias y bandas parenquimatosas bibasales. TEM 1-2 con lesiones líticas pequeñas en mayoría de vértebras dorsolumbares, sin afectar discos intervertebrales ni canal medular. En hemicuerpos vertebrales antero-izquierdos (D12-S1), 4 lesiones líticas en promedio, con reacción esclerótica regenerativa circundante; discretas lesiones líticas en arco posterior, apófisis trasversas, espinosas y huesos iliacos; absceso frío pre y circunvertebral. Radiografía de cráneo A-P y lateral mostró múltiples lesiones osteolíticas en sacabocado.

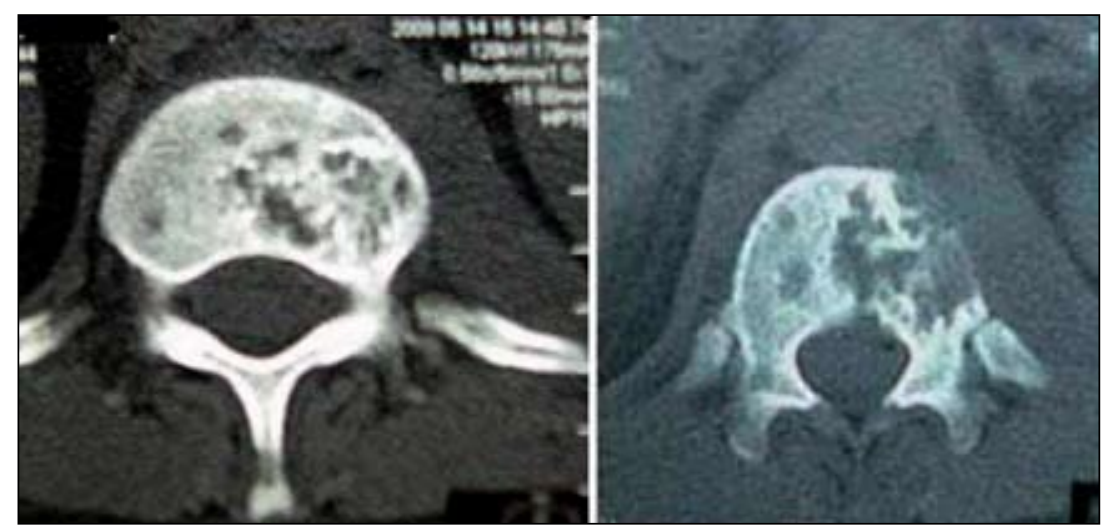

Foto 4. A la izquierda, múltiples lesiones líticas en L1. A la derecha, D12 con lesiones líticas y reborde esclerótico; absceso frío para y prevertebral; fístula en área anterior de médula espinal. 


\section{DISCUSIÓN}

Aunque el paciente negativizó sus BAARs en esputo a fines de 2008, no hubo mejoría frente al tratamiento antituberculoso (Esquema 1:2RHZE/4HR2), cuya prueba de proporciones inicial demostró pansensibilidad. Posteriormente, al identificarse en el curso del tratamiento una prueba de sensibilidad MODS ${ }^{(11)}$, con resistencia a RFP, pero sensible a $\mathrm{H} / \mathrm{ET} / \mathrm{PZ} / \mathrm{S}$, se diagnosticó fracaso del esquema 1 , por respuesta clínica inadecuada, debido a reinfección TBC. Al posibilitarse la expresión del curso natural de la TBC en inmunosuprimidos, se comprometió pulmón, ganglios linfáticos ${ }^{(10-14)}$, tejido subcutáneo del codo, parrilla costal y otros, diseminándose por vía hematógena a áreas de gran concentración de tejido hematopoyético, como hueso esponjoso, diploe craneal ${ }^{(10,15-17)}$. Como las lesiones líticas craneales eran múltiples y con aspecto de sacabocado, fue obligatorio descartar mieloma múltiple ${ }^{(18)}$ y otras neoplasias ${ }^{(19)}$.

La ausencia de componente monoclonal (en suero y orina) y de plasmoblastos en médula ósea, aunada a la presencia de lesiones óseas regenerativas adyacentes a las líticas y abscesos fríos, descartó mieloma múltiple. Las metástasis cancerosas por linfomas no Hodgkin raramente comprometen vértebras o pedículos contiguos. En general, las metástasis casi siempre muestran espacios discales preservados, sin abscesos prevertebrales ${ }^{(16)}$. Ayudan al diagnóstico diferencial final la adecuada respuesta clínica del paciente a la terapia anti-MDR-TB y las características imagenológicas de las lesiones óseas. Las lesiones osteolíticas inducidas por mieloma múltiple son circulares y circunscritas, rodeadas de osteopenia, no exhibiendo trayectos fistulosos, ni osteoesclerosis tempranas ${ }^{(17)}$, excepto después de largo tiempo de tratamiento exitoso.

La tuberculosis craneal en inmunocompetentes exhibe cierto patrón fisiopatológico; las lesiones osteolíticas comprometen usualmente una a dos áreas, dada la restricción a la diseminación ósea del bacilo por los granulomas competentes ${ }^{(12)}$. Lo contrario ocurre en coinfecciones TBC-VIH, cuando el afectado tiene tendencia a desarrollar tuberculosis ósea sistémica multifocal $^{(6,8)}$, con diseminación hematógena extensa y presencia de granulomas óseos incompletos ${ }^{(20)}$; se produce gran número de lesiones craneales, osteítis, abscesos fríos, balonamientos indoloros fronto-esfenoidales-temporo-occipitales del cuero cabelludo, trayectos fistulosos en huesos del diploe o en vértebras dorsolumbares contiguas, secuestros óseos y colecciones abscedadas, preferentemente entre los 3 y 30 años, con el $90 \%$ de hallazgos que ocurren en menores de 20 años ${ }^{(8)}$.

La toma sagital de las últimas vértebras dorso-lumbares de este paciente mostró destrucción ósea, osteosclerosis reparativa y múltiples fístulas anteroizquierdas, por donde se expulsaba material necrótico y caseum (abscesos fríos). La esclerosis ósea, rara al inicio, puede indicar infección secundaria. La evidencia radiológica de curación puede ser vista después de dos meses de tratamiento, con formación de nuevo hueso en los bordes ${ }^{(1)}$. Se nota los abscesos por delante y circundando las vértebras lumbares ${ }^{(21-25)}$. La parte posterior del cuerpo de D12-L1, muestra compresión de la zona anterior del canal vertebral, condicionando paraparesia.

Tanto en cráneo como en las vértebras de este paciente se distinguió tres tipos de lesiones líticas: a) circunscritas con márgenes mal definidos ${ }^{\left({ }^{8}\right)}$; b) en expansión; y, c) con bordes escleróticos -índice de recuperación-, en tanto el paciente había recibido 3 meses de terapia anti-MDRTB ${ }^{(26)}$.

Un patrón frecuente de tuberculosis vertebral es el constituido por lesiones paradiscales adyacentes al cuerpo vertebral, condicionantes de estrechamientos de espacios discales por afectación directa o destrucción del hueso subcondral, con herniación discal al interior del cuerpo vertebral (27).

Como lo refrenda la literatura, el tratamiento de coinfecciones HIV/ MDR-TB debe incluir un mínimo de seis medicamentos, por un periodo de 18 a 24 meses ${ }^{(28)}$.

Concluimos que, en este paciente en tratamiento por infección por $\mathrm{VIH}$, se produjo una reinfección MDR-TB contraída hospitalariamente (o en la comunidad), demostrada por diferentes pruebas de sensibilidad. La proclividad a reinfecciones podría haber sido condicionada por el particular estado de respuesta inmune de este paciente (CD4\% $\mathrm{VL}^{+}$: respuesta virológica exclusiva), expresada en respuestas discordantes al Targa ${ }^{(29)}$. Los cultivos reportados, los hallazgos clínicos sistémicos, la excelente respuesta al tratamiento anti-MDR-TB y las características de las lesiones osteolíticas respaldan el diagnóstico de una TBC sistémica, incluida la ósea, en coinfección con $\mathrm{VIH}^{(30)}$.

\section{Agradecimientos:}

Al Jefe de Servicio de Neumología del Hospital Nacional Dos de Mayo, Dr. Alfonso Uribe Barreto.

\section{REFERENCIAS BIBLIOGRÁFICAS}

1. Awasthy N, Chand K, Singh A. Calvarial tuberculosis: Review of six cases. Ann Indian Acad Neurol. 2006;9:227-9.

2. Capdevila 0, Zurita A, Domingo E, Corbella $X$, Alcaide F, Monfort IL, Femandez-Viladrich P. Multiple cranial osteolytic lesions due to Mycobacterium kansasii in a patient with AIDS. Scand J Infect Dis. 1998;30(3):305-6.

3. Jadhav RN, Palande DA. Calvarial tuberculosis. Neurosurgery. 1999;45(6):1345.

4. Rajesh A, Purohit AK, Lakshmi V. Calvarial tubercular osteomyelitic abscess. Indian J Med Microbiol. 2009;27:380-1.

5. Luzzati R, Bobicchio P, Shah D, Franchi F, Gregori M, Tacconi L. Calvarial tuberculosis as the initial presentation of AIDS. J Clin Neurosci. 2006; 13(6):675-7.

6. Tripathi AK, Gupta N, Khanna M, Ahmad R, Tripathi P. Tuberculosis presenting as osteolytic soft tissue swellings of skull in HIV positive patient: A case report. Indian J Tuberc. 2007;54:193-5.

7. Ramdurg SR, Gupta DK, Suri A, Sharma BS, Mahapatra AK. Calvarial tuberculosis: Uncommon manifestation of common disease--a series of 21 cases. Br J Neurosurg. 2010;24 (5):572-7.

8. Raut AA, Nagar AM, Muzumdar D, Chawla AJ, Narlawar RS, Fattepurkar $S$, et al. Imaging features of calvarial tuberculosis: A study of 42 cases. Am J Neuroradiol. 2004;25:409-14.

9. Mukherjee KK, Kaushik R, Nada R, Khosla VK, Khandelwal N, Kak VK. Calvarial tuberculosis. Surgical Neurol. 2002;57(3):195-202.

10. Diyora B, Kumar R, Modgi R, Sharma A. Calvarial tuberculosis: A report of eleven patients. Neurol India. 2009;57:607-12. 
11. Moore DA, Evans CA, Gilman RH, Caviedes L, Coronel J, Vivar A, et al. Microscopic-observation drug-susceptibility assay for the diagnosis of TB. NEJM. 2006;355(15):1539-50.

12. Ngilimana PJ, Metz T, Munyantore S, Mureganshuro JM , Noël H, Roels H . Lymph node tuberculosis in HIV-1 seropositive patients in Central Africa. A characteristic histopathologic picture. Ann Pathol. 1995;15(1):38-44.

13. Hochedez P, Zeller V, Truffot C, Ansart S, Caumes E, Tubiana R, Katlama C, Bricaire F, Bossi P. Lymphnode tuberculosis in patients infected or not with HIV: general characteristics, clinical presentation, microbiological diagnosis and treatment. PatholBiol. 2003;51(8-9):496-502.

14. Perenboom RM, Richter C, Swai ABM, Kitinya J, Mtoni I, Chande H, Kazema RR. Clinical features of HIV seropositive and HIV seronegative patients with tuberculous lymphadenitis in Dar es Salaam. Tuber Lung Dis. 1995; 76(5):401-6.

15. Prinsloo JG, Kirsten GF. Tuberculosis of the skull vault. S Afr Med J. 1977;51:248.

16. Assadi M, Nabipour I, Eftekhari M, Ebrahimi A, Abotorab SR, Salimipour H, Javadi H, Vahdat K, Ghasemikhah R, Saghari M. Diagnostic role of whole body bone scintigraphy in atypical skeletal tuberculosis resembling multiple metastases: a case report. J Med Case Reports. 2009;3:141-4.

17. Khan SA, Zahid M, Sharmaand B, Hasan AS. Tuberculosis of frontal bone: A case report. Ind J Tub. 2001;48:95.
18.Starcich R. Histopathological and microradiographical observations on myelomatous osteopathy. Acta Haematol. 1957;18(2):113-25.

19. Khattry N, Thulkar S, Das A, Khan SA, Bakshi S. Spinal tuberculosis mimicking malignancy: atypical images features. Indian J Pediat. 2007;74(3):297-8.

20. Soriano V, Tor J. Multifocal bone tuberculosis in one AIDS patient. Genitourin Med. 1992;68:273-6.

21.Danzinger J, Bloch S, Cremi BJ, Goldblatt M. Cranial and intracranial tuberculosis. S Afr Med J. 1976;50:1403.

22. Nergizoĝlu G, Duman N, Ertürk S, Keven K, Ateş $\mathrm{K}, \mathrm{Akar} \mathrm{H}$, et al. Tuberculosis of the skull in a patient on maintenance haemodialysis. Nephrol Dial Transplant. 1999;14:2019-21.

23. Stones DK, Schoeman CJ. Calvarial tuberculosis. J Trop Ped. 2004;50(6):361-4.

24. Chauhan A, Gupta BB, Manohar R. Spinal tuberculous. J Indian Acad Clin Med. 2007;8(1):110-4.

25. Kanabar P. Tuberculosis of lumbar spine. Indian J Orthop. 2005;39:81-9.

26. Sterling TR, Lehmann HP, Frieden TR. Impact of DOTS compared with DOTS-Plus on multidrug resistant tuberculosis and tuberculosis deaths: decision analysis. BMJ. 2003;326(7389):574.

27. Iseman MD. Treatment of multidrug-resistant tuberculosis. NEJM. 1993;329(11):784-91.

28. Gandhi NR, Shah NS, Andrews JR, Vella V, Moll AP, Scott M, Weissman D, Marra C, Lalloo UG,
Friedland GH. HIV coinfection in multidrug- and extensively drug-resistant tuberculosis results in high early mortality. Am J Resp Crit Care Med. 2010;181:80-6.

29. Tan R, Westfall AO, Willig JH, Mugavero MJ, Saag MS, Kaslow RA, Kempf MC. Clinical outcome of HIV-infected antiretroviral-naive patients with discordant immunologic and virologic responses to highly active antiretroviral therapy. J Acquir Immune Defic Syndr. 2008;47:553-8.

30. Fisk TL, Hon HM, Lennox IL, Fordham von Reyn C, Horsburgh CR Jr, et al. Detection of latent tuberculosis among HIV infected patients after initiation of highly active anti-retroviral therapy. AIDS. 2003;12:1102-4.

Manuscrito recibido el 18 de setiembre y aceptado para publicación el 29 de setiembre de 2010.

Correspondencia:

Dr. Víctor Mechán Méndez

Departamento de Medicina

Hospital Nacional Dos de Mayo

Av. Grau s/n

Correo electrónicol: victor.mechan@gmail.com 